\title{
Macdonald operators at infinity
}

\author{
M.L. Nazarov • E.K. Sklyanin
}

Received: 19 September 2012 / Accepted: 7 September 2013 / Published online: 27 September 2013

(C) Springer Science+Business Media New York 2013

\begin{abstract}
We construct a family of pairwise commuting operators such that the Macdonald symmetric functions of infinitely many variables $x_{1}, x_{2}, \ldots$ and of two parameters $q, t$ are their eigenfunctions. These operators are defined as limits at $N \rightarrow \infty$ of renormalized Macdonald operators acting on symmetric polynomials in the variables $x_{1}, \ldots, x_{N}$. They are differential operators in terms of the power sum variables $p_{n}=x_{1}^{n}+x_{2}^{n}+\cdots$ and we compute their symbols by using the Macdonald reproducing kernel. We express these symbols in terms of the Hall-Littlewood symmetric functions of the variables $x_{1}, x_{2}, \ldots$ Our result also yields elementary step operators for the Macdonald symmetric functions.
\end{abstract}

Keywords Macdonald symmetric functions

\section{Introduction}

Over the last two decades the Macdonald polynomials [13] were the subject of much attention in Combinatorics and Representation Theory. These polynomials are symmetric in the $N$ variables $x_{1}, \ldots, x_{N}$ and also depend on two parameters denoted by $q$ and $t$. They are labeled by partitions of $0,1,2, \ldots$ with no more than $N$ parts. Up to normalization, they can be defined as eigenfunctions of certain linear operators acting on the space of all symmetric polynomials in the variables $x_{1}, \ldots, x_{N}$ with coefficients from the field $\mathbb{Q}(q, t)$. These operators have been introduced by Macdonald [13] as the coefficients of a certain operator valued polynomial $D_{N}(u)$ of degree $N$ in a variable $u$ with the constant term 1, see (2.16). In particular, Macdonald has observed that all the eigenvalues of the coefficient of $D_{N}(u)$ at $u$ are already free

M.L. Nazarov · E.K. Sklyanin

Department of Mathematics, University of York, York YO10 5DD, UK 
from multiplicities. Hence this operator coefficient alone can be used to define the Macdonald polynomials.

It is quite common in Combinatorics to extend various symmetric polynomials to an infinite countable set of variables. In particular, the Macdonald polynomials are extended to infinitely many variables $x_{1}, x_{2}, \ldots$ by using the stability property (2.18) of these polynomials and by passing to their limits as $N \rightarrow \infty$. The limits are the Macdonald symmetric functions, which are labeled by partitions of $0,1,2, \ldots$ They have been also studied very well. In particular, the limit at $N \rightarrow \infty$ of a renormalized coefficient of the $D_{N}(u)$ at $u$ was considered in [13]. Another expression for the same limit was given in [2], see also [3, 6].

The limits at $N \rightarrow \infty$ of other coefficients of the $D_{N}(u)$ have so far received less attention. However, in the remarkable work of Shiraishi [18] the limits of certain linear combinations of all the coefficients were expressed in terms of the vertex operators associated with an infinite dimensional Heisenberg Lie algebra, see also $[5,20]$. In a more recent work [1] this result of [18] has been reformulated by using the well-known correspondence [7] between the vertex operators and the HallLittlewood symmetric functions, which are specializations of the Macdonald symmetric functions at $q=0$.

In the present article we consider the limits at $N \rightarrow \infty$ of linear combinations of all the coefficients of $D_{N}(u)$, different from those in $[1,5,18,20]$. Our linear combinations arise naturally from the theory of the double affine Hecke algebras, see for instance [17]. We also express our limits in terms of the Hall-Littlewood symmetric functions. Once stated our result can be derived from those of $[1,18]$. However, we obtained our result independently. Moreover, our proof is different and yields new identities related to the Hall-Littlewood polynomials. Again, the latter can be regarded as specializations of the Macdonald polynomials at $q=0$.

The Macdonald polynomials can be regarded as generalizations of the Jack polynomials which are symmetric in $x_{1}, \ldots, x_{N}$ and also depend on a formal parameter $\alpha$. The latter polynomials are obtained from the former when $q=t^{\alpha}$ and $t \rightarrow 1$. Then the coefficients of $D_{N}(u)$ degenerate to the Sekiguchi-Debiard differential operators $[4,16]$. In [15] we studied the limits of the latter operators at $N \rightarrow \infty$. In the present article we generalize the main result of that work to the Macdonald case. However, the methods used here and in [15] are quite different.

As an application of our result, we construct elementary step operators for the Macdonald symmetric functions. In terms of the labels, our operators correspond to decreasing any given non-zero part of a partition by 1 and to the operation on partitions inverse to that, see our formulas (2.42) and (2.40) respectively. For the origins of this construction see the work [19] and references therein. For related but different results on the Macdonald polynomials see the works [9, 10] and [11].

Here is the plan of the present article. In Sect. 2 we recall some basic facts from the theory of symmetric functions, including the definition of Macdonald polynomials. After establishing the basics we state our main result, which is an explicit expression for the limit of a renormalized polynomial $D_{N}(u)$ at $N \rightarrow \infty$. Then we explicitly construct our elementary step operators. The proof of our main result is given in Sect. 3. Our main tool is the notion of the symbol of an operator relative to the reproducing kernel associated with the Macdonald polynomials. Using it, we reduce the 
proof to a certain determinantal identity for each $N=1,2, \ldots$ which is proved in the rest of Sect. 3.

In this article we generally keep to the notation of the book [13] for symmetric functions. When using results from [13] we simply indicate their numbers within the book. For example, the statement (6.9) from Chap. I of the book will be referred to as [I.6.9] assuming it is from [13]. We do not number our own lemmas, propositions, theorems or corollaries because here we have only one of each.

\section{Symmetric functions}

\subsection{Monomial functions and power sums}

Fix a field $\mathbb{F}$. For any integer $N \geq 1$ denote by $\Lambda_{N}$ the $\mathbb{F}$-algebra of symmetric polynomials in $N$ variables $x_{1}, \ldots, x_{N}$. The algebra $\Lambda_{N}$ is graded by the polynomial degree. The substitution $x_{N}=0$ defines a homomorphism $\Lambda_{N} \rightarrow \Lambda_{N-1}$ preserving the degree. Here $\Lambda_{0}=\mathbb{F}$. The inverse limit of the sequence

$$
\Lambda_{1} \leftarrow \Lambda_{2} \leftarrow \cdots
$$

in the category of graded algebras is denoted by $\Lambda$. The elements of $\Lambda$ are called symmetric functions. Following [13] we will introduce some standard bases of $\Lambda$.

Let $\lambda=\left(\lambda_{1}, \lambda_{2}, \ldots\right)$ be any partition of $0,1,2, \ldots$ The number of non-zero parts is called the length of $\lambda$ and is denoted by $\ell(\lambda)$. If $\ell(\lambda) \leq N$ then the sum of all distinct monomials obtained by permuting the $N$ variables in $x_{1}^{\lambda_{1}} \cdots x_{N}^{\lambda_{N}}$ is denoted by $m_{\lambda}\left(x_{1}, \ldots, x_{N}\right)$. The symmetric polynomials $m_{\lambda}\left(x_{1}, \ldots, x_{N}\right)$ with $\ell(\lambda) \leq N$ form a basis of the vector space $\Lambda_{N}$. By definition, for $\ell(\lambda) \leq N$,

$$
m_{\lambda}\left(x_{1}, \ldots, x_{N}\right)=\sum_{1 \leq i_{1}<\cdots<i_{k} \leq N} \sum_{\sigma \in \mathfrak{S}_{k}} c_{\lambda}^{-1} x_{i_{\sigma(1)}}^{\lambda_{1}} \cdots x_{i_{\sigma(k)}}^{\lambda_{k}},
$$

where we write $k$ instead of $\ell(\lambda)$. Here $\mathfrak{S}_{k}$ is the symmetric group permuting the numbers $1, \ldots, k$ and

$$
c_{\lambda}=k_{1} ! k_{2} ! \cdots
$$

if $k_{1}, k_{2}, \ldots$ are the respective multiplicities of the parts $1,2, \ldots$ of $\lambda$. Further,

$$
m_{\lambda}\left(x_{1}, \ldots, x_{N-1}, 0\right)= \begin{cases}m_{\lambda}\left(x_{1}, \ldots, x_{N-1}\right) & \text { if } \ell(\lambda)<N \\ 0 & \text { if } \ell(\lambda)=N\end{cases}
$$

Hence for any fixed partition $\lambda$ the sequence of polynomials $m_{\lambda}\left(x_{1}, \ldots, x_{N}\right)$ with $N \geq \ell(\lambda)$ has a limit in $\Lambda$. This limit is called the monomial symmetric function corresponding to $\lambda$. Simply omitting the variables, we will denote the limit by $m_{\lambda}$. With $\lambda$ ranging over all partitions of $0,1,2, \ldots$ the symmetric functions $m_{\lambda}$ form a basis of the vector space $\Lambda$. Note that if $\ell(\lambda)=0$ then we set $m_{\lambda}=1$.

We will be also using another standard basis of the vector space $\Lambda$. For each $n=1,2, \ldots$ denote $p_{n}\left(x_{1}, \ldots, x_{N}\right)=x_{1}^{n}+\cdots+x_{N}^{n}$. When the index $n$ is fixed the 
sequence of symmetric polynomials $p_{n}\left(x_{1}, \ldots, x_{N}\right)$ with $N=1,2, \ldots$ has a limit in $\Lambda$, called the power sum symmetric function of degree $n$. We will denote it by $p_{n}$. More generally, for any partition $\lambda$, put

$$
p_{\lambda}=p_{\lambda_{1}} p_{\lambda_{2}} \cdots,
$$

where we set $p_{0}=1$. The elements $p_{\lambda}$ form another basis of $\Lambda$. In other words, the elements $p_{1}, p_{2}, \ldots$ are free generators of the commutative algebra $\Lambda$ over $\mathbb{F}$.

In this article we will be using the natural ordering of partitions. By definition, here $\lambda \geq \mu$ if $\lambda$ and $\mu$ are partitions of the same number and

$$
\lambda_{1} \geq \mu_{1}, \quad \lambda_{1}+\lambda_{2} \geq \mu_{1}+\mu_{2}, \quad \ldots .
$$

This is a partial ordering. Note that by [I.6.9] any monomial symmetric function $m_{\mu}$ is a linear combination of the symmetric functions $p_{\lambda}$ where $\lambda \geq \mu$.

\subsection{Hall-Littlewood functions}

Choose $\mathbb{F}$ to be the field $\mathbb{Q}(t)$ where $t$ is a formal parameter. Take any partition $\lambda$ with $\ell(\lambda) \leq N$. Using the notation of (2.1), put

$$
v_{\lambda}(t)=\prod_{i \geq 0} \prod_{j=1}^{k_{i}} \frac{1-t^{j}}{1-t}
$$

where $k_{0}=N-\ell(\lambda)$. Consider the sum of all the $N$ ! products obtained from

$$
x_{1}^{\lambda_{1}} \cdots x_{N}^{\lambda_{N}} \prod_{1 \leq i<j \leq N} \frac{x_{i}-t x_{j}}{x_{i}-x_{j}}
$$

by permuting $x_{1}, \ldots, x_{N}$. This sum is a symmetric polynomial in $x_{1}, \ldots, x_{N}$ with coefficients from $\mathbb{Z}[t]$. Dividing it by $v_{\lambda}(t)$ we get the Hall-Littlewood symmetric polynomial $P_{\lambda}\left(x_{1}, \ldots, x_{N}\right)$, see [III.2.1]. All coefficients of the latter polynomial also belong to $\mathbb{Z}[t]$ by [III.1.5]. Furthermore, by [III.2.5] similarly to (2.2) we have

$$
P_{\lambda}\left(x_{1}, \ldots, x_{N-1}, 0\right)= \begin{cases}P_{\lambda}\left(x_{1}, \ldots, x_{N-1}\right) & \text { if } \ell(\lambda)<N \\ 0 & \text { if } \ell(\lambda)=N .\end{cases}
$$

Hence for any fixed partition $\lambda$ the sequence of polynomials $P_{\lambda}\left(x_{1}, \ldots, x_{N}\right)$ with $N \geq \ell(\lambda)$ has a limit in $\Lambda$. This is the Hall-Littlewood symmetric function $P_{\lambda}$.

Along with the symmetric function $P_{\lambda}$ it is convenient to use the symmetric function $Q_{\lambda}$ which is a scalar multiple of $P_{\lambda}$. By definition,

$$
Q_{\lambda}=b_{\lambda}(t) P_{\lambda}
$$

where

$$
b_{\lambda}(t)=\prod_{i \geq 1} \prod_{j=1}^{k_{i}}\left(1-t^{j}\right)
$$


We will also use the symmetric polynomial

$$
Q_{\lambda}\left(x_{1}, \ldots, x_{N}\right)=b_{\lambda}(t) P_{\lambda}\left(x_{1}, \ldots, x_{N}\right) .
$$

When we need to distinguish $x_{1}, x_{2}, \ldots$ from any other variables, we will write $f\left(x_{1}, x_{2}, \ldots\right)$ instead of $f \in \Lambda$. Now let $y_{1}, y_{2}, \ldots$ be variables independent of the $x_{1}, x_{2}, \ldots$ Then by [III.4.4] we have the identity

$$
\prod_{i, j=1}^{\infty} \frac{1-t x_{i} y_{j}}{1-x_{i} y_{j}}=\sum_{\lambda} Q_{\lambda}\left(x_{1}, x_{2}, \ldots\right) P_{\lambda}\left(y_{1}, y_{2}, \ldots\right),
$$

where $\lambda$ ranges over all partitions of $0,1,2, \ldots$ The product at the left-hand side of this identity is regarded as an infinite sum of monomials in $x_{1}, x_{2}, \ldots$ and in $y_{1}, y_{2}, \ldots$ by expanding the factor corresponding to $i, j$ as a series at $x_{i} y_{j} \rightarrow 0$.

Note that at $t=0$ both $P_{\lambda}\left(x_{1}, \ldots, x_{N}\right)$ and $Q_{\lambda}\left(x_{1}, \ldots, x_{N}\right)$ specialize to the Schur symmetric polynomial $s_{\lambda}\left(x_{1}, \ldots, x_{N}\right)$. Respectively, the symmetric functions $P_{\lambda}$ and $Q_{\lambda}$ specialize at $t=0$ to the Schur symmetric function $s_{\lambda}$. The symmetric function $P_{\lambda}$ also admits specialization at $t=1$. By [III.2.4] the latter specialization coincides with the monomial symmetric function $m_{\lambda}$.

Now take the symmetric function $Q_{\lambda}$ corresponding to the partition $\lambda=(n)$ with one part only. We will denote this symmetric function by $Q_{n}$. By using a variable $u$ independent of $x_{1}, x_{2}, \ldots$ and $t$ introduce the generating function

$$
Q(u)=1+\sum_{n=1}^{\infty} Q_{n} u^{n}
$$

By [III.2.10] then

$$
Q(u)=\prod_{i=1}^{\infty} \frac{1-t x_{i} u}{1-x_{i} u} .
$$

By taking the logarithm of the infinite product here and then exponentiating,

$$
Q(u)=\exp \left(\sum_{n=1}^{\infty} \frac{1-t^{n}}{n} p_{n} u^{n}\right) .
$$

\subsection{Green polynomials}

The basis of Hall-Littlewood symmetric functions can be related to the basis of $p_{\lambda}$ as follows. Write

$$
p_{\lambda}=\sum_{\mu} X_{\lambda \mu}(t) P_{\mu}
$$

where $X_{\lambda \mu}(t) \in \mathbb{Q}(t)$ while both $\lambda$ and $\mu$ are partitions of the same number. By [III.2.7] each $X_{\lambda \mu}(t)$ is a polynomial in the variable $t$ with integral coefficients. Furthermore, by [III.7.7] this polynomial in $t$ is monic and has the degree

$$
n_{\mu}=\sum_{i \geq 1}(i-1) \mu_{i}
$$


The elements

$$
t^{n_{\mu}} X_{\lambda \mu}\left(t^{-1}\right) \in \mathbb{Z}[t]
$$

are called the Green polynomials, see [Ex. III.7.7] and references therein.

Since $P_{\mu}$ specializes at $t=0$ to the Schur symmetric function $s_{\mu}$, the value $X_{\lambda \mu}(0)$ coincides with the value of the irreducible character of the symmetric group labeled by the partition $\mu$ at the conjugacy class labeled by the partition $\lambda$. Moreover, there are orthogonality relations [III.7.3]:

$$
\sum_{\lambda} X_{\lambda \mu}(t) X_{\lambda \nu}(t) / z_{\lambda}(t)=\delta_{\mu \nu} b_{\mu}(t)
$$

where

$$
z_{\lambda}(t)=z_{\lambda} \prod_{i=1}^{\ell(\lambda)} \frac{1}{1-t^{\lambda_{i}}}
$$

while

$$
z_{\lambda}=1^{k_{1}} k_{1} ! 2^{k_{2}} k_{2} ! \cdots
$$

in the notation of (2.1). At $t=0$ the relations (2.12) specialize to the standard orthogonality relations for the irreducible characters of symmetric groups. Due to (2.5) and to (2.12) the definition (2.11) of the polynomials $X_{\lambda \mu}(t)$ implies that

$$
Q_{\mu}=\sum_{\lambda} X_{\lambda \mu}(t) p_{\lambda} / z_{\lambda}(t) .
$$

\subsection{Macdonald functions}

Now let $\mathbb{F}$ be the field $\mathbb{Q}(q, t)$ where $q$ and $t$ are formal parameters independent of each other. Define a bilinear form $\langle$,$\rangle on the vector space \Lambda$ by setting, for any $\lambda$ and $\mu$,

$$
\left\langle p_{\lambda}, p_{\mu}\right\rangle=z_{\lambda} \delta_{\lambda \mu} \prod_{i=1}^{\ell(\lambda)} \frac{1-q^{\lambda_{i}}}{1-t^{\lambda_{i}}}
$$

in the notation of (2.13). This form is obviously symmetric and non-degenerate. By [VI.4.7] there exists a unique family of elements $M_{\lambda} \in \Lambda$ such that

$$
\left\langle M_{\lambda}, M_{\mu}\right\rangle=0 \quad \text { for } \lambda \neq \mu
$$

and such that any $M_{\lambda}$ equals $m_{\lambda}$ plus a linear combination of the elements $m_{\mu}$ with $\mu<\lambda$ in the natural partial ordering. The elements $M_{\lambda} \in \Lambda$ are called the Macdonald symmetric functions. Alternatively, they can be defined as follows.

Take the algebra $\Lambda_{N}$ of symmetric polynomials in the variables $x_{1}, \ldots, x_{N}$. For each index $i=1, \ldots, N$ define the $q$-shift operator $T_{i}$ on the algebra $\Lambda_{N}$ by

$$
\left(T_{i} f\right)\left(x_{1}, \ldots, x_{N}\right)=f\left(x_{1}, \ldots, q x_{i}, \ldots, x_{N}\right) .
$$


Denote by $\Delta\left(x_{1}, \ldots, x_{N}\right)$ the Vandermonde polynomial of $N$ variables

$$
\operatorname{det}\left[x_{i}^{N-j}\right]_{i, j=1}^{N}=\prod_{1 \leq i<j \leq N}\left(x_{i}-x_{j}\right)
$$

Put

$$
D_{N}(u)=\Delta\left(x_{1}, \ldots, x_{N}\right)^{-1} \cdot \operatorname{det}\left[x_{i}^{N-j}\left(1-u t^{1-j} T_{i}\right)\right]_{i, j=1}^{N},
$$

where $u$ is another variable. The last determinant is defined as the alternated sum

$$
\sum_{\sigma \in \mathfrak{S}_{N}}(-1)^{\sigma} \prod_{i=1}^{N}\left(x_{i}^{N-\sigma(i)}\left(1-u t^{1-\sigma(i)} T_{i}\right)\right)
$$

where, as usual, $(-1)^{\sigma}$ denotes the sign of permutation $\sigma$. In every product over $i=1, \ldots, N$ appearing in (2.17) the operator factors pairwise commute, hence their ordering does not matter. By [VI.4.16] the $D_{N}(u)$ is a polynomial in $u$ with pairwise commuting operator coefficients preserving the space $\Lambda_{N}$. We will call the restrictions of the coefficients to the space $\Lambda_{N}$ the Macdonald operators. By [VI.4.15] they have a common eigenbasis in $\Lambda_{N}$ parameterized by partitions $\lambda$ of length $\ell(\lambda) \leq N$. These eigenvectors are the Macdonald symmetric polynomials.

For each $\lambda$ with $\ell(\lambda) \leq N$ there is an eigenvector denoted by $M_{\lambda}\left(x_{1}, \ldots, x_{N}\right)$ which is equal to $m_{\lambda}\left(x_{1}, \ldots, x_{N}\right)$ plus a linear combination of the polynomials $m_{\mu}\left(x_{1}, \ldots, x_{N}\right)$ with $\mu<\lambda$ and $\ell(\mu) \leq N$. It turns out that each coefficient in this linear combination does not depend on $N$. Note that if $\lambda$ and $\mu$ are any two partitions of the same number such that $\lambda \geq \mu$, then $\ell(\lambda) \leq \ell(\mu)$ by [I.1.11]. It follows that the polynomials $M_{\lambda}\left(x_{1}, \ldots, x_{N}\right)$ have the same stability property as the polynomials $m_{\lambda}\left(x_{1}, \ldots, x_{N}\right)$ in $(2.2)$ :

$$
M_{\lambda}\left(x_{1}, \ldots, x_{N-1}, 0\right)= \begin{cases}M_{\lambda}\left(x_{1}, \ldots, x_{N-1}\right) & \text { if } \ell(\lambda)<N \\ 0 & \text { if } \ell(\lambda)=N\end{cases}
$$

In particular, the sequence of polynomials $M_{\lambda}\left(x_{1}, \ldots, x_{N}\right)$ with $N \geq \ell(\lambda)$ has a limit in $\Lambda$. This is exactly the Macdonald symmetric function $M_{\lambda}$. Further, the eigenvalues of Macdonald operators acting on $\Lambda_{N}$ are known. By [VI.4.15],

$$
D_{N}(u) M_{\lambda}\left(x_{1}, \ldots, x_{N}\right)=\prod_{i=1}^{N}\left(1-u q^{\lambda_{i}} t^{1-i}\right) \cdot M_{\lambda}\left(x_{1}, \ldots, x_{N}\right) .
$$

Note that $M_{\lambda}\left(x_{1}, \ldots, x_{N}\right)$ is a homogeneous polynomial of degree $\lambda_{1}+\lambda_{2}+\cdots$,

$$
T_{1} \cdots T_{N} M_{\lambda}\left(x_{1}, \ldots, x_{N}\right)=q^{\lambda_{1}+\lambda_{2}+\cdots} M_{\lambda}\left(x_{1}, \ldots, x_{N}\right) .
$$

Hence the operator $T_{1} \cdots T_{N}$ on $\Lambda_{N}$ commutes with every coefficient of $D_{N}(u)$. Also note that by [VI.4.14] the symmetric function $M_{\lambda}$ admits a specialization at $q=0$. This specialization equals the Hall-Littlewood symmetric function $P_{\lambda}$. 


\subsection{Reproducing kernel}

In this subsection we will regard the elements of $\Lambda$ as infinite sums of finite products of the variables $x_{1}, x_{2}, \ldots$ For instance, we have

$$
p_{n}=x_{1}^{n}+x_{2}^{n}+\cdots
$$

for any $n \geq 1$. Like in the identity (2.7), we will write $f\left(x_{1}, x_{2}, \ldots\right)$ instead of any $f \in$ $\Lambda$ when we need to distinguish $x_{1}, x_{2}, \ldots$ from other variables. Now let $y_{1}, y_{2}, \ldots$ be variables independent of $x_{1}, x_{2}, \ldots$ According to [VI.2.7], with the bilinear form (2.15) on $\Lambda$ one associates the reproducing kernel

$$
\Pi=\prod_{i, j=1}^{\infty} \frac{\left(t x_{i} y_{j} ; q\right)_{\infty}}{\left(x_{i} y_{j} ; q\right)_{\infty}}
$$

where, as usual,

$$
(u ; q)_{\infty}=\prod_{k=0}^{\infty}\left(1-u q^{k}\right) .
$$

The property of $\Pi$ most useful for us can be stated as the following lemma. For any $f \in \Lambda$ denote by $f^{*}$ the operator on $\Lambda$ adjoint to the multiplication by $f$ relative to the bilinear form (2.15). Note that here $f=f\left(x_{1}, x_{2}, \ldots\right)$.

Lemma We have

$$
f^{*}(\Pi) / \Pi=f\left(y_{1}, y_{2}, \ldots\right) .
$$

Proof The commutative algebra $\Lambda$ is generated by the elements $p_{n}$ with $n \geq 1$. Hence it suffices to prove the lemma for $f=p_{n}$ only. Take the operator $\partial / \partial p_{n}$ of derivation in $\Lambda$ relative to $p_{n}=p_{n}\left(x_{1}, x_{2}, \ldots\right)$. Then, by the definition (2.15),

$$
p_{n}^{*}=n \frac{1-q^{n}}{1-t^{n}} \frac{\partial}{\partial p_{n}} .
$$

On the other hand, by taking the logarithm of (2.21) and then exponentiating,

$$
\Pi=\exp \left(\sum_{n=1}^{\infty} \frac{1}{n} \frac{1-t^{n}}{1-q^{n}} p_{n}\left(x_{1}, x_{2}, \ldots\right) p_{n}\left(y_{1}, y_{2}, \ldots\right)\right) .
$$

The lemma for $f=p_{n}$ follows from the last two displayed equalities.

\subsection{Limits of Macdonald operators}

Let $\mathbb{F}=\mathbb{Q}(q, t)$ as in the two subsections above. For every $N \geq 1$ let $\rho_{N}$ be the homomorphism $\Lambda_{N} \rightarrow \Lambda_{N-1}$ defined by setting $x_{N}=0$, as in the beginning of Sect. 2.1. Denote

$$
A_{N}(u)=\left(T_{1} \cdots T_{N}\right)^{-1} D_{N}(u) /\left(u ; t^{-1}\right)_{N}
$$


where, as usual,

$$
(u ; q)_{N}=\prod_{k=0}^{N-1}\left(1-u q^{k}\right) .
$$

The right-hand side of the equation (2.24) is regarded as a rational function of $u$ with the values being the operators acting on the vector space $\Lambda_{N}$. Due to the stability property (2.18) of the Macdonald polynomials, (2.19) and (2.20) imply

$$
\rho_{N} A_{N}(u)=A_{N-1}(u) \rho_{N}
$$

where $A_{0}(u)=1$. So the sequence of $A_{N}(u)$ with $N \geq 1$ has a limit at $N \rightarrow \infty$. This limit can be written as a series

$$
A(u)=1+A^{(1)} /\left(u ; t^{-1}\right)_{1}+A^{(2)} /\left(u ; t^{-1}\right)_{2}+\cdots
$$

where the leading term equals 1 by (2.20) while the coefficients $A^{(1)}, A^{(2)}, \ldots$ are certain linear operators acting on $\Lambda$. The Macdonald symmetric functions are joint eigenvectors of these operators. Namely, by (2.19) we have the equality

$$
A(u) M_{\lambda}=M_{\lambda} \prod_{i=1}^{\infty} \frac{q^{-\lambda_{i}}-u t^{1-i}}{1-u t^{1-i}} .
$$

In particular, the operators $A^{(1)}, A^{(2)}, \ldots$ pairwise commute and are self-adjoint relative to the bilinear form (2.15). We call them the Macdonald operators at infinity. Due to (2.18) their definition immediately implies that

$$
A^{(k)} M_{\lambda}=0 \quad \text { if } \ell(\lambda)<k .
$$

The operator $A^{(1)}$ has been well studied, see for instance [VI.4.3]. It follows from (2.19) and (2.20) that for any partition $\lambda$,

$$
A^{(1)} M_{\lambda}=\sum_{i=1}^{\infty}\left(q^{-\lambda_{i}}-1\right) t^{i-1} \cdot M_{\lambda} .
$$

In particular, all the eigenvalues of the operator $A^{(1)}$ on $\Lambda$ are pairwise distinct. By [2, Eq. 32] the operator $A^{(1)}$ is equal to the coefficient at 1 of the series in $u$ :

$$
\frac{1}{1-t} \exp \left(\sum_{n=1}^{\infty} \frac{1-t^{n}}{n} u^{n} p_{n}\right) \exp \left(\sum_{n=1}^{\infty}\left(q^{-n}-1\right) u^{-n} \frac{\partial}{\partial p_{n}}\right)-\frac{1}{1-t} .
$$

In the next section we will prove the following general expression for every $A^{(k)}$.

Theorem In the notation (2.5) for every $k=1,2, \ldots$ we have

$$
A^{(k)}=\sum_{\ell(\lambda)=k} q^{-\lambda_{1}-\lambda_{2}-\cdots} Q_{\lambda} P_{\lambda}^{*}
$$

where $\lambda$ ranges over all partitions of length $k$. 
By using (2.5),(2.14) the symmetric functions $P_{\lambda}$ and $Q_{\lambda}$ can be expressed as linear combinations of the functions $p_{\mu}$ where both $\lambda$ and $\mu$ are partitions of the same number. By substituting into (2.26) and then using (2.3),(2.23) one can express every operator $A^{(k)}$ in terms of $p_{n}$ and $\partial / \partial p_{n}$ where $n=1,2, \ldots$

In the case $k=1$ one can also employ the generating function (2.8). In this case by using the equality (2.10), our theorem follows from the expression for the operator $A^{(1)}$ given just before stating the theorem. Furthermore, for any $k \geq 1$ one can derive our theorem from the results of [1, Sect. 3] and [18, Sect. 9]. In the present article we give a proof independent of these results. In particular, our proof yields new identities for the Hall-Littlewood symmetric polynomials.

\subsection{Step operators}

In this subsection we will obtain a corollary to our theorem. We will also utilize the following particular case of the Pieri rule for Macdonald symmetric functions. By [VI.6.24] for any partition $\mu$ the product $p_{1} M_{\mu}$ equals the linear combination of the symmetric functions $M_{\lambda}$ with the coefficients

$$
\prod_{j=1}^{i-1} \frac{1-q^{\lambda_{j}-\lambda_{i}} t^{i-j+1}}{1-q^{\lambda_{j}-\lambda_{i}+1} t^{i-j}} \cdot \prod_{j=1}^{i-1} \frac{1-q^{\lambda_{j}-\lambda_{i}+1} t^{i-j-1}}{1-q^{\lambda_{j}-\lambda_{i}} t^{i-j}}
$$

where $\lambda$ ranges over all partitions such that the sequence $\lambda_{1}, \lambda_{2}, \ldots$ is obtained from $\mu_{1}, \mu_{2}, \ldots$ by increasing one of its terms by 1 and $i$ is the index of the term.

Further, by [VI.6.19] the above stated equality implies that for any partition $\lambda$ the symmetric function $\partial M_{\lambda} / \partial p_{1}=p_{1}^{*} M_{\lambda}(1-t) /(1-q)$ is equal to the linear combination of the $M_{\mu}$ with the coefficients

$$
\prod_{j=1}^{\lambda_{i}-1} \frac{1-q^{\lambda_{i}-j-1} t^{\lambda_{j}^{\prime}-i+1}}{1-q^{\lambda_{i}-j} t^{\lambda_{j}^{\prime}-i}} \cdot \prod_{j=1}^{\lambda_{i}-1} \frac{1-q^{\lambda_{i}-j+1} t^{\lambda_{j}^{\prime}-i}}{1-q^{\lambda_{i}-j} t^{\lambda_{j}^{\prime}-i+1}},
$$

where $\mu$ ranges over all partitions such that the sequence $\mu_{1}, \mu_{2}, \ldots$ is obtained from $\lambda_{1}, \lambda_{2}, \ldots$ by decreasing one of its terms by 1 and $i$ is the index of the term. As usual, here $\lambda^{\prime}=\left(\lambda_{1}^{\prime}, \lambda_{2}^{\prime}, \ldots\right)$ is the partition conjugate to $\lambda$.

Let us now define the linear operators $B^{(1)}, B^{(2)}, \ldots$ acting on $\Lambda$ by setting

$$
\left[p_{1}, A(u)\right]_{q}=-u B(u)(1-q) /(1-t)
$$

where

$$
B(u)=B^{(1)} /\left(u ; t^{-1}\right)_{1}+B^{(2)} /\left(u ; t^{-1}\right)_{2}+\cdots .
$$

At the left-hand side of (2.29) we have the $q$-commutator $p_{1} A(u)-q A(u) p_{1}$.

Further, define the linear operators $C^{(1)}, C^{(2)}, \ldots$ acting on $\Lambda$ by setting

$$
\left[A(u), \partial / \partial p_{1}\right]_{q}=-u C(u),
$$


where

$$
C(u)=C^{(1)} /\left(u ; t^{-1}\right)_{1}+C^{(2)} /\left(u ; t^{-1}\right)_{2}+\cdots .
$$

Then by the definitions of $B(u)$ and $C(u)$ and by (2.23) we have the relation

$$
B(u)^{*}=C(u) .
$$

Our theorem provides explicit expressions for the operators $B^{(1)}, B^{(2)}, \ldots$ and $C^{(1)}, C^{(2)}, \ldots$ which we state as the following corollary. The corollary will allow to explicitly construct the elementary step operators for Macdonald symmetric functions, see the equalities (2.40) and (2.42) below.

Corollary For every $k=0,1,2, \ldots$ we have the equalities

$$
\begin{aligned}
& B^{(k+1)}=t^{-k} \sum_{\ell(\mu)=k} q^{-\mu_{1}-\mu_{2}-\cdots} Q_{\mu \sqcup 1} P_{\mu}^{*}, \\
& C^{(k+1)}=t^{-k} \sum_{\ell(\mu)=k} q^{-\mu_{1}-\mu_{2}-\cdots} P_{\mu} Q_{\mu \sqcup 1}^{*},
\end{aligned}
$$

where $\mu$ ranges over all partitions of length $k$ and $\mu \sqcup 1$ denotes the partition obtained from $\mu$ by appending one extra part 1.

Proof The stated equalities (2.32) and (2.33) follow from each other due to the relation (2.31). We shall derive the first of the two equalities from our theorem.

Recall that at $q=0$ the Macdonald symmetric function $M_{\lambda}$ specializes to the HallLittlewood symmetric function $P_{\lambda}$. Hence the expression for $\partial M_{\lambda} / \partial p_{1}$ given above implies

$$
\partial P_{\lambda} / \partial p_{1}=\sum_{\mu} \psi_{\lambda \mu}(t) P_{\mu}
$$

where $\mu$ ranges over all partitions such that the sequence $\mu_{1}, \mu_{2}, \ldots$ is obtained from $\lambda_{1}, \lambda_{2}, \ldots$ by decreasing one of its terms by 1 . Let $i$ is the index of that term. If $\lambda_{i}=1$ then the coefficient (2.28) is 1 . Then $\psi_{\lambda \mu}(t)=1$ in particular. If $\lambda_{i}>1$ and $q=0$ then the only factor in the two products over the indices $j$ in (2.28) comes from the first product and corresponds to $j=\lambda_{i}-1=\mu_{i}$. Then $\psi_{\lambda \mu}(t)=1-t^{m}$ where $m=\lambda_{j}^{\prime}-i+1$ is the multiplicity of the part $\mu_{i}$ in $\mu$. But we will not use any explicit expression for the coefficient $\psi_{\lambda \mu}(t)$ with $\lambda_{i}>1$.

By [III.4.9] the equality (2.34) established above is equivalent to the equality

$$
(1-t) p_{1} Q_{\mu}=\sum_{\lambda} \psi_{\lambda \mu}(t) Q_{\lambda}
$$

where $\lambda$ ranges over all partitions such that the sequence $\lambda_{1}, \lambda_{2}, \ldots$ is obtained from $\mu_{1}, \mu_{2}, \ldots$ by increasing one of its terms by 1 . The latter equality can also be derived from the multiplication formula (3.7) below, by setting $n=1$ there. 
Now for any $k \geq 1$ consider the $q$-commutator $\left[p_{1}, A(u)\right]_{q}$. By (2.26),

$$
\begin{aligned}
p_{1} A^{(k)}(1-t) & =\sum_{\ell(\mu)=k} q^{-\mu_{1}-\mu_{2}-\cdots}(1-t) p_{1} Q_{\mu} P_{\mu}^{*} \\
& =\sum_{\ell(\mu)=k} \sum_{\lambda} q^{-\mu_{1}-\mu_{2}-\cdots} \psi_{\lambda \mu}(t) Q_{\lambda} P_{\mu}^{*},
\end{aligned}
$$

where we use the notation of (2.35). Further, by (2.26) and (2.34),

$$
\begin{aligned}
q\left[A^{(k)}, p_{1}\right](1-t) /(1-q) & =\sum_{\ell(\lambda)=k} q^{1-\lambda_{1}-\lambda_{2}-\cdots} Q_{\lambda}\left[P_{\lambda}^{*}, p_{1}\right](1-t) /(1-q) \\
& =\sum_{\ell(\lambda)=k} q^{1-\lambda_{1}-\lambda_{2}-\cdots} Q_{\lambda}\left[p_{1}^{*}, P_{\lambda}\right]^{*}(1-t) /(1-q) \\
& =\sum_{\ell(\lambda)=k} q^{1-\lambda_{1}-\lambda_{2}-\cdots} Q_{\lambda}\left(\partial P_{\lambda} / \partial p_{1}\right)^{*} \\
& =\sum_{\ell(\lambda)=k} \sum_{\mu} q^{-\mu_{1}-\mu_{2}-\cdots} \psi_{\lambda \mu}(t) Q_{\lambda} P_{\mu}^{*},
\end{aligned}
$$

where the square brackets stand for the usual operator commutator. Hence

$$
\begin{gathered}
{\left[p_{1}, A(u)\right]_{q}(1-t) /(1-q)=p_{1} A^{(k)}(1-t)-q\left[A^{(k)}, p_{1}\right](1-t) /(1-q)} \\
=\sum_{\ell(\mu)=k} q^{-\mu_{1}-\mu_{2}-\cdots} Q_{\mu \sqcup 1} P_{\mu}^{*}-\sum_{\ell(\mu)=k-1} q^{-\mu_{1}-\mu_{2}-\cdots} Q_{\mu \sqcup 1} P_{\mu}^{*},
\end{gathered}
$$

where we use the equality $\varphi_{\mu \sqcup 1, \mu}(t)=1$. The definition (2.29) now implies that

$$
\begin{aligned}
-u B(u)= & (1-t) p_{1}+\sum_{k=1}^{\infty} \sum_{\ell(\mu)=k} q^{-\mu_{1}-\mu_{2}-\cdots} Q_{\mu \sqcup 1} P_{\mu}^{*} /\left(u ; t^{-1}\right)_{k} \\
& -\sum_{k=1}^{\infty} \sum_{\ell(\mu)=k-1} q^{-\mu_{1}-\mu_{2}-\cdots} Q_{\mu \sqcup 1} P_{\mu}^{*} /\left(u ; t^{-1}\right)_{k} \\
= & \sum_{k=0}^{\infty} \sum_{\ell(\mu)=k} q^{-\mu_{1}-\mu_{2}-\cdots} Q_{\mu \sqcup 1} P_{\mu}^{*} /\left(u ; t^{-1}\right)_{k} \\
& -\sum_{k=0}^{\infty} \sum_{\ell(\mu)=k} q^{-\mu_{1}-\mu_{2}-\cdots} Q_{\mu \sqcup 1} P_{\mu}^{*} /\left(u ; t^{-1}\right)_{k+1} .
\end{aligned}
$$

The required equality (2.32) now follows from the relation

$$
\left(u ; t^{-1}\right)_{k}^{-1}-\left(u ; t^{-1}\right)_{k+1}^{-1}=-u t^{-k}\left(u ; t^{-1}\right)_{k+1}^{-1} .
$$

Note that in the infinite product over the indices $i$ at the right-hand side of the equality (2.25) the only factors different from 1 are those corresponding to 
$i=1, \ldots, \ell(\lambda)$. For any such index $i$ consider the product

$$
\frac{t^{1-i}}{1-u t^{1-i}} \prod_{\substack{j=1 \\ j \neq i}}^{\ell(\lambda)} \frac{q^{-\lambda_{j}}-u t^{1-j}}{1-u t^{1-j}} .
$$

It follows from (2.25) and from the definition (2.29) that, for any given partition $\mu$,

$$
B(u) M_{\mu}=\sum_{\lambda} B_{\lambda \mu}(u) M_{\lambda}
$$

where $B_{\lambda \mu}(u)$ equals the product of (2.27) by (2.37) and by $1-t$, while $\lambda$ ranges over all partitions such that the sequence $\lambda_{1}, \lambda_{2}, \ldots$ is obtained from $\mu_{1}, \mu_{2}, \ldots$ by increasing one of its terms by 1 and $i$ is the index of the term.

Similarly, (2.25) and (2.30) imply that for any given $\lambda$,

$$
C(u) M_{\lambda}=\sum_{\mu} C_{\mu \lambda}(u) M_{\mu},
$$

where $C_{\mu \lambda}(u)$ equals the product of (2.28) by (2.37) and by $1-q$, while $\mu$ ranges over all partitions such that the sequence $\mu_{1}, \mu_{2}, \ldots$ is obtained from $\lambda_{1}, \lambda_{2}, \ldots$ by decreasing one of its terms by 1 and $i$ is the index of the term.

Now let the partition $\lambda$ be fixed. Then for the indices $i=1, \ldots, \ell(\lambda)$ all the elements $q^{-\lambda_{i}} t^{i-1}$ of the field $\mathbb{Q}(q, t)$ are pairwise distinct. Therefore by (2.38) for the partition $\mu$ corresponding to any of these indices $i$ we have

$$
B\left(q^{-\lambda_{i}} t^{i-1}\right) M_{\mu}=B_{\lambda \mu}\left(q^{-\lambda_{i}} t^{i-1}\right) M_{\lambda},
$$

where the coefficient $B_{\lambda \mu}\left(q^{-\lambda_{i}} t^{i-1}\right)$ is the product of (2.27) by $1-t$ and by

$$
t^{1-i} \prod_{j=1}^{\ell(\lambda)} \frac{1}{q^{\lambda_{i}}-t^{i-j}} \prod_{\substack{j=1 \\ j \neq i}}^{\ell(\lambda)}\left(q^{\lambda_{i}-\lambda_{j}}-t^{i-j}\right) .
$$

The left-hand side of the equality (2.40) should be understood as the value in $\Lambda$ of the rational function $B(u) M_{\mu}$ at the point $u=q^{-\lambda_{i}} t^{i-1}$. Similarly, by (2.39),

$$
C\left(q^{-\lambda_{i}} t^{i-1}\right) M_{\lambda}=C_{\mu \lambda}\left(q^{-\lambda_{i}} t^{i-1}\right) M_{\mu},
$$

where $C_{\mu \lambda}\left(q^{-\lambda_{i}} t^{i-1}\right)$ is the product of (2.28) by $1-q$ and by (2.41).

Our definitions (2.29) and (2.30) of the series $B(u)$ and $C(u)$ are motivated by the results from [17, Sect. 1]. But our definitions employ the $q$-commutators of the operators $p_{1}$ and $\partial / \partial p_{1}$ with $A(u)$, while in [17] the usual commutators have been employed. Our theorem also provides analogues of the equalities (2.32) and (2.33) for the usual commutators of $p_{1}$ and $\partial / \partial p_{1}$ with $A(u)$. These analogues however involve summation over the pairs of partitions $\lambda$ and $\mu$, see (2.36) above. 


\section{Proof of the theorem}

\subsection{Reduction of the proof}

In this section we will reduce the proof of our theorem to a certain determinantal identity for each $N=1,2, \ldots$. By the lemma from Sect. 2.5 the theorem is equivalent to the equality

$$
A(u)(\Pi) / \Pi=\sum_{\lambda} q^{-\lambda_{1}-\lambda_{2}-\cdots} Q_{\lambda}\left(x_{1}, x_{2}, \ldots\right) P_{\lambda}\left(y_{1}, y_{2}, \ldots\right) /\left(u ; t^{-1}\right)_{\ell(\lambda)},
$$

where the coefficients of the series $A(u)$ are regarded as operators acting on the symmetric functions in the variables $x_{1}, x_{2}, \ldots$. Here we let the $\lambda$ range over all partitions of $0,1,2, \ldots$ and assume that $\left(u ; t^{-1}\right)_{0}=1$.

It suffices to prove for $N=1,2, \ldots$ the restriction of the required functional equality to

$$
x_{N+1}=x_{N+2}=\cdots=0 .
$$

By the definition of $A(u)$ the restriction of $A(u)(\Pi) / \Pi$ to (3.1) as of a function in $x_{1}, x_{2}, \ldots$ equals

$$
A_{N}(u)\left(\Pi_{N}\right) / \Pi_{N},
$$

where we denote

$$
\Pi_{N}=\prod_{i=1}^{N} \prod_{j=1}^{\infty} \frac{\left(t x_{i} y_{j} ; q\right)_{\infty}}{\left(x_{i} y_{j} ; q\right)_{\infty}}
$$

By the definition of the symmetric function $Q_{\lambda}\left(x_{1}, x_{2}, \ldots\right)$ its restriction to (3.1) is $Q_{\lambda}\left(x_{1}, \ldots, x_{N}\right)$ if $\ell(\lambda) \leq N$ and vanishes if $\ell(\lambda)>N$. Hence the restriction of the right-hand side of the required functional equality to (3.1) is

$$
\sum_{\ell(\lambda) \leq N} q^{-\lambda_{1}-\lambda_{2}-\cdots} Q_{\lambda}\left(x_{1}, \ldots, x_{N}\right) P_{\lambda}\left(y_{1}, y_{2}, \ldots\right) /\left(u ; t^{-1}\right)_{\ell(\lambda)} .
$$

Due to [VI.2.19] to prove the equality between (3.2) and (3.3) it suffices to set

$$
y_{N+1}=y_{N+2}=\cdots=0 \text {. }
$$

However, we will keep working with the infinite collection of variables $y_{1}, y_{2}, \ldots$. This will simplify the induction argument in the next subsection. Note that by replacing in (3.3) each variable $x_{i}$ with $q x_{i}$ we get a sum independent of $q$ :

$$
\sum_{\ell(\lambda) \leq N} Q_{\lambda}\left(x_{1}, \ldots, x_{N}\right) P_{\lambda}\left(y_{1}, y_{2}, \ldots\right) /\left(u ; t^{-1}\right)_{\ell(\lambda)} .
$$

Let us compute the function (3.2). This function depends on the variable $u$ rationally. It is also symmetric in either of the two collections of variables $x_{1}, \ldots, x_{N}$ and 
$y_{1}, y_{2}, \ldots$ It can be obtained by applying to the identity function 1 the result of conjugating $A_{N}(u)$ by the operator of multiplication by $\Pi_{N}$, see also $[9$, Sect. 1] for a similar argument. By the definition (2.16) we have

$$
\left(T_{1} \cdots T_{N}\right)^{-1} D_{N}(u)=\Delta\left(x_{1}, \ldots, x_{N}\right)^{-1} \cdot \operatorname{det}\left[x_{i}^{N-j}\left(T_{i}^{-1}-u t^{1-j}\right)\right]_{i, j=1}^{N} .
$$

The last determinant is defined as the alternated sum

$$
\sum_{\sigma \in \mathfrak{S}_{N}}(-1)^{\sigma} \prod_{i=1}^{N}\left(x_{i}^{N-\sigma(i)}\left(T_{i}^{-1}-u t^{1-\sigma(i)}\right)\right)
$$

Conjugating this sum by $\Pi_{N}$ amounts to replacing every $T_{i}^{-1}$ by its conjugate

$$
\prod_{l=1}^{\infty} \frac{1-q^{-1} t x_{i} y_{l}}{1-q^{-1} x_{i} y_{l}} \cdot T_{i}^{-1}
$$

see the definition (2.22). Hence we get the sum

$$
\sum_{\sigma \in \mathfrak{S}_{N}}(-1)^{\sigma} \prod_{i=1}^{N}\left(x_{i}^{N-\sigma(i)}\left(\prod_{l=1}^{\infty} \frac{1-q^{-1} t x_{i} y_{l}}{1-q^{-1} x_{i} y_{l}} \cdot T_{i}^{-1}-u t^{1-\sigma(i)}\right)\right) .
$$

Here in any single summand each of the factors corresponding to $i=1, \ldots, N$ does not depend on the variables $x_{j}$ with $j \neq i$. Therefore applying the latter operator sum to 1 amounts to simply deleting each $T_{i}^{-1}$. Thus we get the function

$$
\begin{gathered}
\sum_{\sigma \in \mathfrak{S}_{N}}(-1)^{\sigma} \prod_{i=1}^{N}\left(x_{i}^{N-\sigma(i)}\left(\prod_{l=1}^{\infty} \frac{1-q^{-1} t x_{i} y_{l}}{1-q^{-1} x_{i} y_{l}}-u t^{1-\sigma(i)}\right)\right) \\
=\operatorname{det}\left[x_{i}^{N-j}\left(\prod_{l=1}^{\infty} \frac{1-q^{-1} t x_{i} y_{l}}{1-q^{-1} x_{i} y_{l}}-u t^{1-j}\right)\right]_{i, j=1}^{N} .
\end{gathered}
$$

Dividing by $\Delta\left(x_{1}, \ldots, x_{N}\right)$ and then replacing each variable $x_{i}$ with $q x_{i}$ we get

$$
\Delta\left(x_{1}, \ldots, x_{N}\right)^{-1} \cdot \operatorname{det}\left[x_{i}^{N-j}\left(\prod_{l=1}^{\infty} \frac{1-t x_{i} y_{l}}{1-x_{i} y_{l}}-u t^{1-j}\right)\right]_{i, j=1}^{N} .
$$

Thus to prove our theorem it suffices to show that for $N=1,2, \ldots$ the sum (3.4) is equal to the ratio (3.5) divided by $\left(u ; t^{-1}\right)_{N}$, see the definition (2.24). In the next subsection we will reduce the proof of this equality to a family of certain identities for symmetric polynomials in the single collection of variables $x_{1}, \ldots, x_{N}$. These identities will correspond to partitions $\lambda$ with $0<\ell(\lambda)<N$. 


\subsection{Further reduction}

Let us consider the last determinant in (3.5). We will be proving by induction on $N$ that this determinant is equal to the sum (3.4) multiplied by the Vandermonde polynomial $\Delta\left(x_{1}, \ldots, x_{N}\right)$ and by $\left(u ; t^{-1}\right)_{N}$.

If $N=0$ then there is only one term in the sum (3.4), and this term is 1 . The leading term of the series $A(u)$ is also 1 . Hence we can use the case $N=0$ as the induction base. Now take any $N \geq 1$ and suppose that the required equality holds for $N-1$ instead of $N$. For each $i=1, \ldots, N$ we will for short denote

$$
\Delta^{(i)}=\Delta\left(x_{1}, \ldots, \widehat{x}_{i}, \ldots, x_{N}\right),
$$

where, as usual, the symbol $\widehat{x}_{i}$ indicates the omitted variable. Similarly, for any partition $\mu$ with $\ell(\mu)<N$ we will for short denote

$$
Q_{\mu}^{(i)}=Q_{\mu}\left(x_{1}, \ldots, \widehat{x}_{i}, \ldots, x_{N}\right) .
$$

Due to (2.9) the infinite product over the index $l$ in (3.5) equals the sum

$$
1+\sum_{n=1}^{\infty} Q_{n}\left(y_{1}, y_{2}, \ldots\right) x_{i}^{n} .
$$

Therefore by expanding the last determinant in (3.5) in its first column and then employing the induction assumption where $u$ and $\lambda$ are replaced with $u t^{-1}$ and $\mu$, respectively, we get the sum

$$
\begin{aligned}
& \sum_{i=1}^{N}(-1)^{i+1} x_{i}^{N-1}\left(1-u+\sum_{n=1}^{\infty} Q_{n}\left(y_{1}, y_{2}, \ldots\right) x_{i}^{n}\right) \Delta^{(i)} \\
& \quad \times \sum_{\ell(\mu)<N} Q_{\mu}^{(i)} P_{\mu}\left(y_{1}, y_{2}, \ldots\right) \prod_{\ell(\mu)<l<N}\left(1-u t^{-l}\right) .
\end{aligned}
$$

Let us open the brackets in the first of the two lines of the display (3.6) and use the multiplication formula due to Morris [14]:

$$
Q_{n}\left(y_{1}, y_{2}, \ldots\right) P_{\mu}\left(y_{1}, y_{2}, \ldots\right)=\sum_{\lambda} \varphi_{\lambda \mu}(t) P_{\lambda}\left(y_{1}, y_{2}, \ldots\right)
$$

see also [III.5.7]. Here $\varphi_{\lambda \mu}(t) \neq 0$ only if

$$
\lambda_{1} \geq \mu_{1} \geq \lambda_{2} \geq \mu_{2} \geq \cdots
$$

and

$$
\lambda_{1}-\mu_{1}+\lambda_{2}-\mu_{2}+\cdots=n .
$$

Then in the notation (2.1) the coefficient $\varphi_{\lambda \mu}(t)$ is the product of the differences $1-t^{k_{i}}$ taken over all the indices $i \geq 1$ such that

$$
\lambda_{i}^{\prime}-\mu_{i}^{\prime}>\lambda_{i+1}^{\prime}-\mu_{i+1}^{\prime}
$$


where $\lambda^{\prime}=\left(\lambda_{1}^{\prime}, \lambda_{2}^{\prime}, \ldots\right)$ and $\mu^{\prime}=\left(\mu_{1}^{\prime}, \mu_{2}^{\prime}, \ldots\right)$ are the conjugate partitions.

In the proof of our theorem we will not use this explicit expression for the coefficient $\varphi_{\lambda \mu}(t)$. We have reproduced it here for the sake of completeness. We will use only the fact that the inequality $\varphi_{\lambda \mu}(t) \neq 0$ implies (3.8) and (3.9). We also note that for any fixed partition $\lambda$ with $\ell(\lambda) \leq N$ and any index $i=1, \ldots, N$ the multiplication formula (3.7) implies the decomposition formula

$$
Q_{\lambda}\left(x_{1}, \ldots, x_{N}\right)=\sum_{\ell(\mu)<N} \varphi_{\lambda \mu}(t) x_{i}^{n} Q_{\mu}^{(i)},
$$

where the sum is taken over all partitions $\mu$ with $\ell(\mu)<N$ while $n$ is determined by the equality (3.9), see for instance [III.5.5] and [III.5.14].

Using the multiplication formula (3.7), the sum (3.6) equals

$$
\begin{aligned}
& \sum_{i=1}^{N} \sum_{\ell(\mu)<N}(-1)^{i+1} x_{i}^{N-1} \Delta^{(i)} Q_{\mu}^{(i)} P_{\mu}\left(y_{1}, y_{2}, \ldots\right)(1-u) \prod_{\ell(\mu)<l<N}\left(1-u t^{-l}\right) \\
& \quad+\sum_{i=1}^{N} \sum_{\ell(\mu)<N} \sum_{n=1}^{\infty}(-1)^{i+1} x_{i}^{N-1+n} \Delta^{(i)} Q_{\mu}^{(i)} \prod_{\ell(\mu)<l<N}\left(1-u t^{-l}\right) \\
& \quad \times \sum_{\lambda} \varphi_{\lambda \mu}(t) P_{\lambda}\left(y_{1}, y_{2}, \ldots\right) .
\end{aligned}
$$

Note that under the condition (3.8) the inequality $n \geq 1$ is equivalent to $\lambda \neq \mu$. Also note that under the condition (3.8) the length $\ell(\lambda)$ is equal to $\ell(\mu)$ or to $\ell(\mu)+1$. In particular, if $\varphi_{\lambda \mu}(t) \neq 0$ in (3.11) then $\ell(\lambda) \leq N$.

Let us now fix any partition $\lambda$ with $\ell(\lambda) \leq N$ and compare the coefficients at $P_{\lambda}\left(y_{1}, y_{2}, \ldots\right)$ in the sum displayed in the three lines (3.11), and in the sum (3.4) multiplied by $\Delta\left(x_{1}, \ldots, x_{N}\right)$ and $\left(u ; t^{-1}\right)_{N}$. The latter coefficient always equals

$$
\Delta\left(x_{1}, \ldots, x_{N}\right) Q_{\lambda}\left(x_{1}, \ldots, x_{N}\right) \prod_{\ell(\lambda) \leq l<N}\left(1-u t^{-l}\right) .
$$

But when taking the coefficient in (3.11) we will separately consider three cases.

First suppose that $\ell(\lambda)=0$. In this case there is no partition $\mu$ satisfying the condition (3.9) with $n \geq 1$. By setting $\ell(\mu)=0$ in the first line of (3.11) we get

$$
\sum_{i=1}^{N}(-1)^{i+1} x_{i}^{N-1} \Delta^{(i)} \prod_{0 \leq l<N}\left(1-u t^{-l}\right),
$$

which equals (3.12) with $\ell(\lambda)=0$. Hence the two coefficients are the same here. Next suppose that $\ell(\lambda)=N$. Then the first line of (3.11) does not contribute to the coefficient at $P_{\lambda}\left(y_{1}, y_{2}, \ldots\right)$ since $\ell(\mu)<N$ in that line. Consider the last two lines of (3.11). If $\varphi_{\lambda \mu}(t) \neq 0$ there then $\ell(\mu)=N-1$ by the condition (3.8), so that the product over $l$ is actually 1 . Then the inequality $n \geq 1$ holds since $\ell(\mu)<\ell(\lambda)$. Thus 
the coefficient at $P_{\lambda}\left(y_{1}, y_{2}, \ldots\right)$ with $\ell(\lambda)=N$ in (3.11) equals

$$
\sum_{i=1}^{N} \sum_{\ell(\mu)<N}(-1)^{i+1} x_{i}^{N-1+n} \Delta^{(i)} Q_{\mu}^{(i)} \varphi_{\lambda \mu}(t) .
$$

Using the decomposition formula (3.10), the last displayed sum equals

$$
\Delta\left(x_{1}, \ldots, x_{N}\right) Q_{\lambda}\left(x_{1}, \ldots, x_{N}\right)
$$

and hence coincides with (3.12) in the case $\ell(\lambda)=N$ under our consideration.

Finally let $0<\ell(\lambda)<N$. Then the coefficient at $P_{\lambda}\left(y_{1}, y_{2}, \ldots\right)$ in (3.11) is

$$
\begin{aligned}
& \sum_{i=1}^{N}(-1)^{i+1} x_{i}^{N-1} \Delta^{(i)} Q_{\lambda}^{(i)}(1-u) \prod_{\ell(\lambda)<l<N}\left(1-u t^{-l}\right) \\
& +\sum_{\substack{i=1 \\
\ell}}^{N} \sum_{\substack{\ell(\mu)<N \\
n \geq 1}}(-1)^{i+1} x_{i}^{N-1+n} \Delta^{(i)} Q_{\mu}^{(i)} \varphi_{\lambda \mu}(t) \prod_{\ell(\mu)<l<N}\left(1-u t^{-l}\right) .
\end{aligned}
$$

The sum displayed in the first of the above two lines can be rewritten as

$$
\begin{aligned}
& \sum_{i=1}^{N}(-1)^{i+1} x_{i}^{N-1} \Delta^{(i)} Q_{\lambda}^{(i)} \prod_{\ell(\lambda) \leq l<N}\left(1-u t^{-l}\right) \\
& \quad+\sum_{i=1}^{N}(-1)^{i+1} x_{i}^{N-1} \Delta^{(i)} Q_{\lambda}^{(i)}\left(u t^{-\ell(\lambda)}-u\right) \prod_{\ell(\lambda)<l<N}\left(1-u t^{-l}\right) .
\end{aligned}
$$

Further, in the second line of the display (3.14) we may have $\varphi_{\lambda \mu}(t) \neq 0$ only if $\ell(\mu)$ equals $\ell(\lambda)$ or $\ell(\lambda)-1$. Therefore the sum in that line can be rewritten as

$$
\begin{aligned}
& \sum_{i=1}^{N} \sum_{\substack{\ell(\mu)=\ell(\lambda) \\
\mu \neq \lambda}}(-1)^{i+1} x_{i}^{N-1+n} \Delta^{(i)} Q_{\mu}^{(i)} \varphi_{\lambda \mu}(t) \prod_{\ell(\lambda) \leq l<N}\left(1-u t^{-l}\right) \\
& \quad+\sum_{\substack{i=1 \\
\ell}}^{N} \sum_{\substack{\mu \neq=\ell \\
\mu \neq \lambda}}(-1)^{i+1} x_{i}^{N-1+n} \Delta^{(i)} Q_{\mu}^{(i)} \varphi_{\lambda \mu}(t) u t^{-\ell(\lambda)} \prod_{\ell(\lambda)<l<N}\left(1-u t^{-l}\right) \\
& \quad+\sum_{i=1}^{N} \sum_{\ell(\mu)<\ell(\lambda)}(-1)^{i+1} x_{i}^{N-1+n} \Delta^{(i)} Q_{\mu}^{(i)} \varphi_{\lambda \mu}(t) \prod_{\ell(\lambda) \leq l<N}\left(1-u t^{-l}\right) .
\end{aligned}
$$

Using the decomposition formula (3.10), the sums displayed in the first line of (3.15) and in the first and the third lines of (3.16) add up to the product (3.12). This product is the coefficient at $P_{\lambda}\left(y_{1}, y_{2}, \ldots\right)$ in the sum (3.4) multiplied by $\Delta\left(x_{1}, \ldots, x_{N}\right)$ and $\left(u ; t^{-1}\right)_{N}$. The sums displayed in the second line of (3.15) and 
in the second line of (3.16) should add up to zero. Let us multiply each of these two sums by $t^{\ell(\lambda)}$ and divide them by their common factors $u$ and

$$
1-u t^{-l} \text { where } 1 \ell(\lambda)<l<N .
$$

The proof of our theorem thus reduces to the next combinatorial proposition.

Proposition For any fixed partition $\lambda$ with $0<\ell(\lambda)<N$ we have the identity

$$
\sum_{i=1}^{N}(-1)^{i+1} x_{i}^{N-1} \Delta^{(i)}\left(Q_{\lambda}^{(i)}\left(1-t^{\ell(\lambda)}\right)+\sum_{\substack{\ell(\mu)=\ell(\lambda) \\ \mu \neq \lambda}} x_{i}^{n} Q_{\mu}^{(i)} \varphi_{\lambda \mu}(t)\right)=0,
$$

where $n$ is determined by the partitions $\lambda$ and $\mu$ via the equality (3.9).

Note that at the left-hand side of the above identity we have a skew-symmetric polynomial in the variables $x_{1}, \ldots, x_{N}$ with the coefficients from $\mathbb{Z}[t]$. Dividing it by the Vandermonde polynomial $\Delta\left(x_{1}, \ldots, x_{N}\right)$ we get a symmetric polynomial in $x_{1}, \ldots, x_{N}$. Our proposition states that the latter polynomial is actually zero.

\subsection{Finishing the proof}

For any non-negative integer $n$ and for any partition $\mu$ with $\ell(\mu)<N$ consider the sum

$$
\sum_{i=1}^{N}(-1)^{i+1} x_{i}^{N-1+n} \Delta^{(i)} Q_{\mu}^{(i)} .
$$

If $n$ is determined by the equality (3.9) for any fixed partition $\lambda$ with $\ell(\lambda)<N$, then the left-hand side of the identity in our proposition is a linear combination of the sums (3.17) with the coefficients $1-t^{\ell(\lambda)}$ or $\varphi_{\lambda \mu}(t)$ if respectively $\mu=\lambda$ or $\mu \neq \lambda$ but $\ell(\mu)=\ell(\lambda)$. Note that $\varphi_{\lambda \lambda}(t)=1$. In particular, $\varphi_{\lambda \lambda}(t) \neq 1-t^{\ell(\lambda)}$.

Denote by $F_{\mu, n}\left(x_{1}, \ldots, x_{N}\right)$ the alternated sum of $N$ ! products obtained from

$$
x_{1}^{\mu_{1}} \cdots x_{N-1}^{\mu_{N-1}} x_{N}^{N-1+n} \prod_{1 \leq i<j<N}\left(x_{i}-t x_{j}\right)
$$

by permuting $x_{1}, \ldots, x_{N}$. Here we use the signs of permutations for alternation. This sum is a skew-symmetric polynomial in $x_{1}, \ldots, x_{N}$ with coefficients from $\mathbb{Z}[t]$. By performing the summation first over the permutations which map $x_{N}$ to $x_{i}$ and then over the indices $i=1, \ldots, N$ one shows that the product

$$
(-1)^{N+1} F_{\mu, n}\left(x_{1}, \ldots, x_{N}\right) \cdot b_{\mu}(t) / v_{\mu}(t)
$$

equals the sum (3.17). Here one uses only the definition of the polynomial $Q_{\mu}^{(i)}$, see the beginning of Sect. 2.2. 
Below we shall prove that if $\mu$ satisfies the conditions $\ell(\mu)=\ell(\lambda)$ and (3.8) while $n$ is determined by (3.9) then $F_{\mu, n}\left(x_{1}, \ldots, x_{N}\right)$ is a linear combination of the products

$$
\Delta\left(x_{1}, \ldots, x_{N}\right) P_{v}\left(x_{1}, \ldots, x_{N}\right),
$$

where $v$ is a partition, $\ell(v) \leq N$ and $v<\lambda$ in the natural ordering. Hence the arguments from the previous two subsections imply that the difference between the leftand right-hand sides of the required equality (2.26) is a linear combination of the operators $P_{v} P_{\lambda}^{*}$ where $v<\lambda$. However, this difference must be self-adjoint relative to the bilinear form (2.15) on the vector space $\Lambda$. Indeed, the left-hand side of (2.26) is self-adjoint by definition, while the right-hand side is self-adjoint due to (2.5). Therefore the difference equals zero.

Now fix any $\mu$ satisfying the conditions $\ell(\mu)=\ell(\lambda)$ and (3.8). Determine the integer $n$ by (3.9). Take any monomial in the variables $x_{1}, \ldots, x_{N}$ resulting from opening the brackets in (3.18). Due to the alternation it suffices to consider only those monomials where $x_{1}, \ldots, x_{N}$ occur with distinct degrees. By rearranging these degrees in the descending order we get a monomial

$$
x_{1}^{\nu_{1}+N-1} \cdots x_{N-1}^{v_{N-1}+1} x_{N}^{\nu_{N}}
$$

where $v_{1} \geq \cdots \geq v_{N} \geq 0$. For any $k=1, \ldots, N-1$ the sum of the first $k$ degrees

$$
\left(v_{1}+N-1\right)+\cdots+\left(v_{k}+N-k\right)
$$

does not exceed the maximum of the following two sums:

$$
\left(\mu_{1}+N-2\right)+\cdots+\left(\mu_{k}+N-k-1\right)
$$

and

$$
\left(\mu_{1}+N-2\right)+\cdots+\left(\mu_{k-1}+N-k\right)+(N-1+n) ;
$$

see the proof of the property [III.2.6] of Hall-Littlewood polynomials for a similar argument. Hence if (3.22) is the maximum of the two sums then due to (3.8),

$$
v_{1}+\cdots+v_{k} \leq \mu_{1}+\cdots+\mu_{k}-k<\lambda_{1}+\cdots+\lambda_{k} .
$$

If (3.23) is the maximum then due to (3.8) and (3.9),

$$
\begin{aligned}
v_{1}+\cdots+v_{k} & \leq \mu_{1}+\cdots+\mu_{k-1}+n \\
& =\left(\lambda_{1}+\cdots+\lambda_{k}\right)-\left(\mu_{k}-\lambda_{k+1}\right)-\cdots-\left(\mu_{N-1}-\lambda_{N}\right) \\
& \leq \lambda_{1}+\cdots+\lambda_{k} .
\end{aligned}
$$

For any $k \leq \ell(\lambda)$ the last inequality is strict because $\ell(\mu)=\ell(\lambda)$. Thus $v<\lambda$.

By the definition of the Schur symmetric polynomial corresponding to the partition $v=\left(v_{1}, \ldots, v_{N}, 0,0, \ldots\right)$ the alternated sum of $N$ ! products obtained by permuting $x_{1}, \ldots, x_{N}$ in the monomial (3.21) is equal to the product

$$
\Delta\left(x_{1}, \ldots, x_{N}\right) s_{v}\left(x_{1}, \ldots, x_{N}\right) .
$$


Hence we have now proved that $F_{\mu, n}\left(x_{1}, \ldots, x_{N}\right)$ is a linear combination of the products (3.24) where $v<\lambda$. In the latter statement, the products (3.24) can be replaced with the respective products (3.20) by using the property [III.2.6].

Thus we have completed the proof of our theorem. Further, by the definitions (2.4) and (2.6) the factor $b_{\mu}(t) / v_{\mu}(t)$ appearing in the product (3.19) equals

$$
(1-t)^{N} \prod_{j=1}^{N-\ell(\mu)}\left(1-t^{j}\right)^{-1}
$$

In particular, this factor is the same for all products (3.19) such that $\ell(\mu)=\ell(\lambda)$. Dividing the identity in our proposition by this factor and by $(-1)^{N+1}$ we get

$$
\left(1-t^{\ell(\lambda)}\right) F_{\lambda, 0}\left(x_{1}, \ldots, x_{N}\right)+\sum_{\substack{\ell(\mu)=\ell(\lambda) \\ \mu \neq \lambda}} \varphi_{\lambda \mu}(t) F_{\mu, n}\left(x_{1}, \ldots, x_{N}\right)=0
$$

for any fixed partition $\lambda$ such that $0<\ell(\lambda)<N$. Here the positive integer $n$ is determined by the partitions $\lambda$ and $\mu$ via the equality (3.9).

It would be interesting to prove this identity without using any properties of Macdonald operators, for instance by employing the methods of [12, Chap. 7]. It would be also interesting to find a link between this identity and that from [8].

Acknowledgements We are grateful to M.V. Feigin, G.I. Olshanski, J. Shiraishi and E. Vasserot for helpful comments on this work. The first and the second named of us have been supported by the EPSRC grants EP/I 014071 and EP/H000054, respectively.

\section{References}

1. Awata, H., Kanno, H.: Macdonald operators and homological invariants of the colored Hopf link. J. Phys. A 44, 375201 (2011)

2. Awata, H., Matsuo, Y., Odake, S., Shiraishi, J.: Collective fields, Calogero-Sutherland model and generalized matrix models. Phys. Lett. B 347, 49-55 (1995)

3. Cai, W., Jing, N.: A generalization of Newton's identity and Macdonald functions. arXiv:1210.1621

4. Debiard, A.: Polynômes de Tchébychev et de Jacobi dans un espace euclidien de dimension $p$. C. R. Acad. Sc. Paris I, Math. 296, 529-532 (1983)

5. Feigin, B., Hashizume, K., Hoshino, A., Shiraishi, J., Yanagida, S.: A commutative algebra on degenerate $\mathbb{C P}^{1}$ and Macdonald polynomials. J. Math. Phys. 50, 095215 (2009)

6. Garsia, A.M., Haiman, M.: A remarkable $q$, $t$-Catalan sequence and $q$-Lagrange inversion. J. Algebr. Comb. 5, 191-244 (1996)

7. Jing, N.: Vertex operators and Hall-Littlewood symmetric functions. Adv. Math. 87, 226-248 (1991)

8. Jing, N.: Quantum Kac-Moody algebras and vertex representations. Lett. Math. Phys. 44, 261-271 (1998)

9. Kirillov, A.N., Noumi, M.: Affine Hecke algebras and raising operators for Macdonald polynomials. Duke Math. J. 93, 1-39 (1998)

10. Kajihara, Y., Noumi, M.: Raising operators of row type for Macdonald polynomials. Compos. Math. 120, 119-136 (2000)

11. Lapointe, L., Vinet, L.: Rodrigues formulas for the Macdonald polynomials. Adv. Math. 130, 261-279 (1997)

12. Lascoux, A.: Symmetric Functions and Combinatorial Operators on Polynomials. American Mathematical Society, Providence (2003) 
13. Macdonald, I.G.: Symmetric Functions and Hall Polynomials. Oxford University Press, London (1995)

14. Morris, A.O.: A note on multiplication of Hall functions. J. Lond. Math. Soc. 39, 481-488 (1964)

15. Nazarov, M.L., Sklyanin, E.K.: Sekiguchi-Debiard operators at infinity. arXiv:1212.2781

16. Sekiguchi, J.: Zonal spherical functions on some symmetric spaces. Publ. Res. Inst. Math. Sci. 12, 455-459 (1977)

17. Schiffmann, O., Vasserot, E.: The elliptic Hall algebra and the equivariant K-theory of the Hilbert scheme of $\mathbb{A}^{2}$. Duke Math. J. 162, 279-366 (2013)

18. Shiraishi, J.: A family of integral transformations and basic hypergeometric series. Commun. Math. Phys. 263, 439-460 (2006)

19. Sklyanin, E.K.: Separation of variables. New trends. Prog. Theor. Phys. Suppl. 118, 35-60 (1995)

20. Yamamoto, T.: Higher order Macdonald operators with stability properties and their applications. Lett. Math. Phys. 77, 169-181 (2006) 